CRYSTALLOGRAPHIC COMMUNICATIONS

ISSN 2056-9890

Received 31 July 2018

Accepted 23 August 2018

Edited by P. Dastidar, Indian Association for the Cultivation of Science, India

Keywords: crystal structure; structure-activity relationships; calcium-channel antagonists; 4aryl-hexahydroquinolines; 1,4-dihydropyridine rings; hydrogen bonding.

CCDC reference: 1863662

Supporting information: this article has supporting information at journals.iucr.org/e

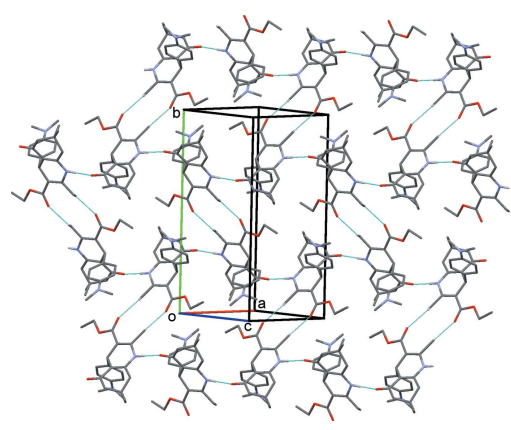

OPEN $\odot$ ACCESS

\section{Crystal structure of ethyl 4-[4-(dimethylamino)- phenyl]-2,7,7-trimethyl-5-oxo-1,4,5,6,7,8-hexa- hydroquinoline-3-carboxylate}

\author{
Scott A. Steiger, ${ }^{a}$ Chun $\mathrm{Li}^{\mathrm{b}}$ and Nicholas R. Natale ${ }^{\mathrm{a} *}$

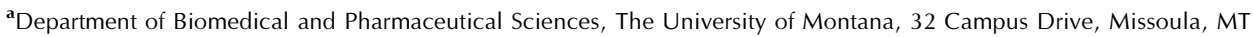 \\ 59812, USA, and bepartment of Chemistry, Ithaca College, 953 Danby Road, Ithaca, NY 14850, USA. \\ *Correspondence e-mail: nicholas.natale@mso.umt.edu
}

In the title racemic compound, ethyl 4-(4-dimethylaminophenyl)-2,7,7trimethyl-5-oxo-1,4,5,6,7,8-hexahydroquinoline-3-carboxylate, the common structural features in this type of compound, such as the flat-boat conformation of the 1,4-dihydropyridine (1,4-DHP) ring, the envelope conformation of the fused cyclohexanone, and the substituted phenyl ring at the pseudo-axial position and orthogonal to the 1,4-DHP ring, are present. In the crystal, molecules are linked via $\mathrm{N}-\mathrm{H} \cdots \mathrm{O}$ and $\mathrm{C}-\mathrm{H} \cdots \mathrm{O}$ hydrogen bonds, forming layers parallel to the $(10 \overline{1})$ plane.

\section{Chemical context}

1,4-Dihydropyridine (DHP) derivatives are well known for their calcium-channel blocking activity and many of these compounds, such as nifedipine, nicardipine, and amlodipine, have been used in the treatment of angina pectoris and systemic hypertension. (Wishart et al., 2006) 4-Aryl-1,4-dihydropyridines that bind the $L$-type voltage-gated calcium channels (VGCC) have been in general medical practice for over three decades (Zamponi, 2005). Many modifications on 1,4-DHP have been performed to obtain active compounds as calcium-channel agonists or antagonists (Martín et al., 1995; Rose, 1990; Rose \& Dräger 1992). One such modifications is fusing a cyclohexanone ring to form hexahydroquinolone, in which the orientation of the carbonyl group of the ester substituent at the 5-position in the 1,4-DHP ring has been fixed. This class of compounds has been shown to have moderate calcium-channel antagonistic activity, as well as antiinflammatory modes and stem-cell differentiation properties, and has been implicated in slowing neurodegenerative disorders (Trippier et al., 2013). Recently, these compounds were found to have distinct selectivity profiles to different calcium channel subtypes (Schaller et al., 2018). Another report also showed that the 4-aryl-hexahydroquinolones, especially the ones containing a methoxy moiety, exhibit good antioxidant property as radical scavengers (Yang et al., 2011). It has been revealed that the aryl group in the 4-position of the 1,4-DHP ring is the basic requirement for optimal activity and the type of electron-withdrawing groups on the phenyl group would affect the receptor-binding activity (Takahashi et al., 2008). It has also been proven that the flattened boat conformation of the 1,4-DHP ring is one factor that leads to higher calciumchannel activity (Linden et al., 2004). In a continuation of our 
study on the structure-activity relationship of this class of 1,4DHP derivatives, i.e. 4-aryl-hexahydroquinolones, we report herein the crystal structure of a compound we synthesized, ethyl 4-(4-dimethylaminophenyl)-2,7,7-trimethyl-5-oxo1,4,5,6,7,8- hexahydroquinoline-3-carboxylate.<smiles>CCOC(=O)C1=C(C)NC2=C(C(=O)CC(C)(C)C2)C1c1ccc(N(C)C)cc1</smiles>

\section{Structural commentary}

The asymmetric unit of the title compound contains one independent molecule crystallizing racemically in the monoclinic space group $P 2_{1} / n$. A displacement ellipsoid plot showing the atomic numbering is presented in Fig. 1.

In the title compound, the 1,4-DHP ring is characterized by a shallow or flattened boat conformation, which is one of the factors that leads to higher calcium-channel activity. The flattened boat conformation is visually obvious with the flat base formed by atoms $\mathrm{C} 2, \mathrm{C} 3, \mathrm{C} 10$, and C9 and the bow and

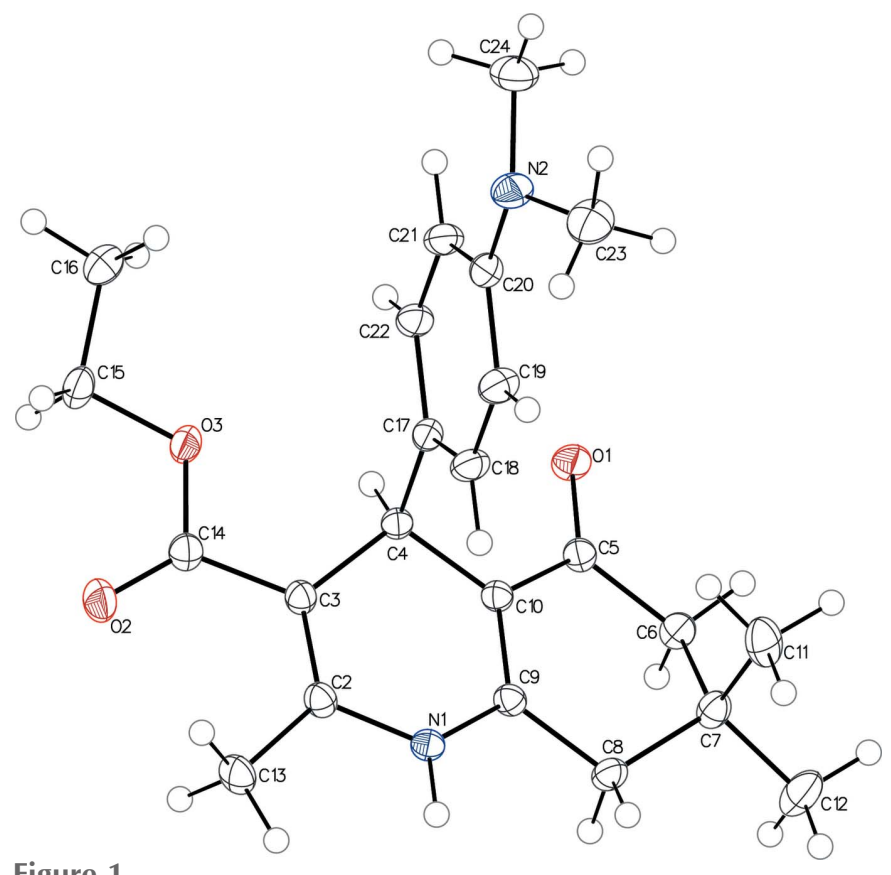

Figure 1

The asymmetric unit of the title compound showing the atom-labeling scheme. Displacement ellipsoids are drawn at the $50 \%$ probability level
Table 1

Hydrogen-bond geometry $\left(\AA{ }^{\circ}\right)$.

\begin{tabular}{lllll}
\hline$D-\mathrm{H} \cdots A$ & $D-\mathrm{H}$ & $\mathrm{H} \cdots A$ & $D \cdots A$ & $D-\mathrm{H} \cdots A$ \\
\hline $\mathrm{N} 1-\mathrm{H} 1 \cdots \mathrm{O} 1^{\mathrm{i}}$ & $0.879(15)$ & $1.968(15)$ & $2.8380(11)$ & $170.6(14)$ \\
$\mathrm{C} 13-\mathrm{H} 13 C \cdots \mathrm{O} 2^{\mathrm{ii}}$ & 0.98 & 2.57 & $3.1741(15)$ & 120 \\
\hline
\end{tabular}

Symmetry codes: (i) $x-\frac{1}{2},-y+\frac{1}{2}, z-\frac{1}{2}$; (ii) $-x,-y+1,-z+1$.

stern formed by the slightly raised $\mathrm{C} 4$ and $\mathrm{N} 1$ atoms. The mean plane defined by atoms $\mathrm{C} 2, \mathrm{C} 3, \mathrm{C} 9$, and $\mathrm{C} 10$ is planar, with an r.m.s. deviation of $0.008 \AA$. The shallowness of the boat conformation is indicated by the marginal displacements of atoms $\mathrm{N} 1[0.1332(15) \AA]$ and $\mathrm{C} 4[0.3047(16) \AA]$ from this mean plane, and is also implied by the small puckering amplitude $Q$ [0.2583 (10) $\AA$ ]

Examination of the fused $\mathrm{C} 5-\mathrm{C} 10$ cyclohexanone ring using puckering parameters also showed that the ring adopts an envelope conformation, with atom $\mathrm{C} 7$ protruding in the same direction as the 4-aryl group at a distance of 0.6453 (15) $\AA$ from the mean plane through the other five $\mathrm{C}$ atoms. The orientation of ring atom $\mathrm{C} 7$ makes the axial bond $\mathrm{C} 7-\mathrm{C} 11$ syn-periplanar to the 4-aryl group, i.e. the torsion angle between $\mathrm{C} 7-\mathrm{C} 11$ and $\mathrm{C} 4-\mathrm{C} 17$ is $6.91(7)^{\circ}$.

The pseudo-axial position of the 4-aryl group is conserved in the title compound. The C17-C22 phenyl ring is almost orthogonal to the base of the 1,4-DHP ring formed by atoms $\mathrm{C} 2$, $\mathrm{C} 3, \mathrm{C} 10$, and $\mathrm{C} 9$, with the dihedral angles between the $\mathrm{C} 2-$ C3-C10-C9 mean plane and the ring being $89.59(3)^{\circ}$. The very small pseudo-torsion angle $\left[2.44(8)^{\circ}\right]$ between $\mathrm{N} 1-\mathrm{C} 4$ and $\mathrm{C} 17-\mathrm{C} 18$ also implies a bisecting orientation of the 4-aryl group to the 1,4-DHP ring in this compound.

As in other 1,4-DHP compounds (Linden et al., 2004), the ester group is coplanar and at a cis orientation to the adjacent endocyclic $\mathrm{C} 2=\mathrm{C} 3$ double bond. The planarity extends out through the ester chains.

The nitrogen atom in the dimethylamino group is almost in the same plane as the phenyl ring, at a distance of 0.0420 (16) $\AA$ from the mean plane. However, the plane formed by N2-C23-C24 is slightly bent from the phenyl group with the angle of $27.24(11)^{\circ}$ rather than being coplanar with the phenyl ring, which seems to be common in $\mathrm{N}, \mathrm{N}$-dimethylaniline type of compounds (Dahl, 2000). In conclusion, the parameters reported here demonstrate that the conformational features usually observed in cyclohexanone-fused 1,4-DHP derivatives have been conserved. As a promising base structure for calcium-channel antagonists, different substitutions and more structural modifications are being carried out in our group. Progress will be reported in due course.

\section{Supramolecular features}

In the crystal, molecules are linked along the diagonal of the ac plane by $\mathrm{N}-\mathrm{H}$.. O hydrogen bonds, forming chains which are in turn linked by $\mathrm{C}-\mathrm{H} \cdots \mathrm{O}$ hydrogen bonds into layers

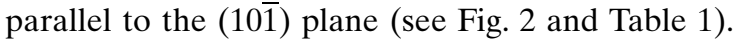




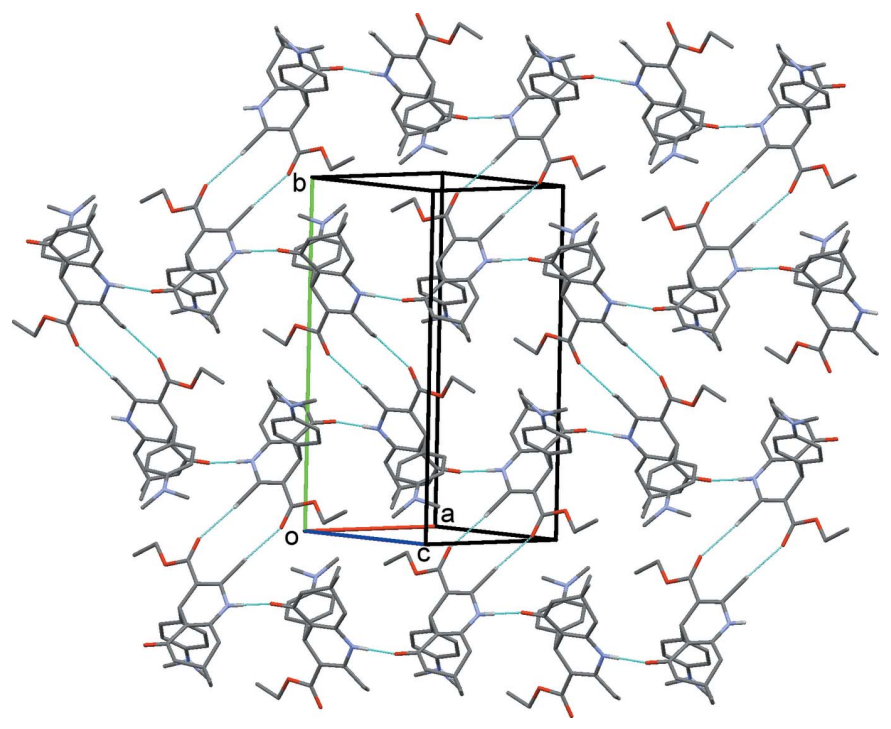

Figure 2

A view normal to plane (10) $)$ of the crystal packing of the title compound. The hydrogen bonds (Table 1) are shown as dashed lines and only $\mathrm{H}$ atoms $\mathrm{H} 1$ and $\mathrm{H} 13 \mathrm{C}$ have been included.

\section{Database survey}

A search in the Cambridge Structural Database (Version 5.39, November 2017) for related compounds with a 4-aryl-hexahydroquinolone-3-carboxylate fragment gave 30 hits. All these compounds share the common structural features such as the flat-boat conformation of the1,4-dihydropyridine (1,4-DHP) ring, the envelope conformation of the fused cyclohexanone ring, and the substituted phenyl ring at the pseudo-axial position and orthogonal to the 1,4-DHP ring.

\section{Synthesis and crystallization}

An oven-dried $100 \mathrm{ml}$ round-bottom flask equipped with a magnetic stir bar was charged with $10 \mathrm{mmol}$ of dimedone, $10 \mathrm{mmol}$ of ethyl acetoacetate and $5 \mathrm{~mol} \%$ of ytterbium(III) trifluoromethanesulfonate. The mixture was then taken up in $30 \mathrm{ml}$ of absolute ethanol, capped and put under an inert atmosphere of argon, after which the solution was allowed to stir at room temperature for $20 \mathrm{~min}$. The appropriate corresponding benzaldehyde $(10 \mathrm{mmol})$ and $10 \mathrm{mmol}$ of ammonium acetate were added to the stirring solution, the solution was allowed to stir at room temperature for $48 \mathrm{~h}$. Reaction progress was monitored via TLC. Once the reaction was complete, excess solvent was removed via rotary evaporation. The solution was then purified via silica column chromatography. The title compound was recrystallized by slow evaporation from hexane and ethyl acetate $(v: v=$ ?:?). m.p. $513.1 \mathrm{~K}$.

${ }^{1} \mathrm{H}$ NMR $\left(\delta, \mathrm{CDCl}_{3}\right)$ p.p.m. $0.95(s, 3 \mathrm{H}), 1.05(s, 3 \mathrm{H}), 1.22(t$, $J=7.08 \mathrm{~Hz}, 3 \mathrm{H}), 2.13 \& 2.19(A B q, 2 \mathrm{H}, J=16.5 \mathrm{~Hz}), 2.17 \&$ $2.27(A B q, 2 \mathrm{H}, J=16.5 \mathrm{~Hz}), 2.85(s, 3 \mathrm{H}), 4.046(q, J=7.08 \mathrm{~Hz}$, $2 \mathrm{H}), 4.94(s, 1 \mathrm{H}), 6.06(s, 1 \mathrm{H}), 6.57(d, J=6.8 \mathrm{~Hz}, 2 \mathrm{H}), 7.14(d$, $J=6.8 \mathrm{~Hz}, 2 \mathrm{H})$.
Table 2

Experimental details.

\begin{tabular}{|c|c|}
\hline \multicolumn{2}{|l|}{ Crystal data } \\
\hline Chemical formula & $\mathrm{C}_{23} \mathrm{H}_{30} \mathrm{~N}_{2} \mathrm{O}_{3}$ \\
\hline$M_{\mathrm{r}}$ & 382.49 \\
\hline Crystal system, space group & Monoclinic, $P 2_{1} / n$ \\
\hline Temperature $(\mathrm{K})$ & 100 \\
\hline$a, b, c(\AA)$ & $9.6834(3), 18.2390(5), 12.2073(3)$ \\
\hline$\beta\left(^{\circ}\right)$ & $105.4464(14)$ \\
\hline$V\left(\AA^{3}\right)$ & $2078.12(10)$ \\
\hline$Z$ & 4 \\
\hline Radiation type & Mo $K \alpha$ \\
\hline$\mu\left(\mathrm{mm}^{-1}\right)$ & 0.08 \\
\hline Crystal size $(\mathrm{mm})$ & $0.46 \times 0.30 \times 0.26$ \\
\hline \multicolumn{2}{|l|}{ Data collection } \\
\hline Diffractometer & Bruker SMART BREEZE CCD \\
\hline Absorption correction & $\begin{array}{l}\text { Multi-scan (SADABS; Bruker, } \\
\text { 2013) }\end{array}$ \\
\hline$T_{\min }, T_{\max }$ & $0.923,1.000$ \\
\hline $\begin{array}{l}\text { No. of measured, independent and } \\
\text { observed }[I>2 \sigma(I)] \text { reflections }\end{array}$ & $48649,7211,5703$ \\
\hline$R_{\text {int }}$ & 0.038 \\
\hline$(\sin \theta / \lambda)_{\max }\left(\AA^{-1}\right)$ & 0.747 \\
\hline \multicolumn{2}{|l|}{ Refinement } \\
\hline$R\left[F^{2}>2 \sigma\left(F^{2}\right)\right], w R\left(F^{2}\right), S$ & $0.049,0.142,1.02$ \\
\hline No. of reflections & 7211 \\
\hline No. of parameters & 264 \\
\hline $\mathrm{H}$-atom treatment & $\begin{array}{l}\mathrm{H} \text { atoms treated by a mixture of } \\
\text { independent and constrained } \\
\text { refinement }\end{array}$ \\
\hline$\Delta \rho_{\max }, \Delta \rho_{\min }\left(\mathrm{e} \AA^{-3}\right)$ & $0.56,-0.21$ \\
\hline
\end{tabular}

Computer programs: APEX2 and SAINT (Bruker, 2013), SHELXS97 (Sheldrick, 2008), SHELXL2014 (Sheldrick, 2015) and OLEX2 (Dolomanov et al., 2009).

${ }^{13} \mathrm{C} \mathrm{NMR}\left(\delta, \mathrm{CDCl}_{3}\right)$ p.p.m. 14.37, 19.55, 27.42, 29.54, 32.82, $35.38,40.86,41.17,50.85,59.84,106.61,112.46,128.70,135.94$, 142.95, 147.85, 149.06, 167.79, 195.75.

MS: calculated for $\mathrm{C}_{23} \mathrm{H}_{30} \mathrm{~N}_{2} \mathrm{O}_{3}, 382.49$; observed: $\mathrm{m} / z=405$ $([M+23] 7), 234(100)$.

\section{Refinement}

Crystal data, data collection and structure refinement details are summarized in Table 2. The $\mathrm{H}$ atoms on methyl groups were constrained to an ideal geometry, with $\mathrm{C}-\mathrm{H}=0.98 \AA$ and $U_{\text {iso }}(\mathrm{H})=1.5 U_{\text {eq }}(\mathrm{C})$, and were allowed to rotate freely about the $\mathrm{C}-\mathrm{C}$ bonds. The rest of the $\mathrm{H}$ atoms were placed in calculated positions with $\mathrm{C}-\mathrm{H}=0.95-1.00 \AA$ and refined as riding on their carrier atoms with $U_{\text {iso }}(\mathrm{H})=1.2 U_{\text {eq }}(\mathrm{C})$. The positions of the amine $\mathrm{H}$ atoms and hydroxyl $\mathrm{H}$ atoms were determined from difference-Fourier maps and freely refined. Three low-angle reflections were omitted from the refinement because their observed intensities were much lower than the calculated values as a result of being partially obscured by the beam stop.

\section{Acknowledgements}

We would like to thank Dr David J. Burkhart of the Center for Translational Medicine at the University of Montana for the mass spectrometry. We thank Dr Robert Zipkin of 
MedChem101 for funds to support the publication costs of this article.

\section{References}

Bruker (2013). APEX2, SAINT and SADABS. Bruker AXS ins., Madison, Wisconsin, USA.

Dahl, T. (2000). Acta Cryst. C56, 708-710.

Dolomanov, O. V., Bourhis, L. J., Gildea, R. J., Howard, J. A. K. \& Puschmann, H. (2009). J. Appl. Cryst. 42, 339-341.

Linden, A., Şafak, C. \& Aydın, F. (2004). Acta Cryst. C60, o711-o713.

Martín, N., Quinteiro, M., Seoane, C., Soto, J., Mora, A., Suárez, M., Ochoa, E., Morales, A. \& Bosque, J. (1995). J. Heterocycl. Chem. 32, 235-238.

Rose, U. (1990). Arch. Pharm. Pharm. Med. Chem. 323, 281-286.

Rose, U. \& Dräger, M. (1992). J. Med. Chem. 35, 2238-2243.
Schaller, D., Gündüz, M. G., Zhang, F. X., Zamponi, G. W. \& Wolber, G. (2018). Eur. J. Med. Chem. 155, 1-12.

Sheldrick, G. M. (2008). Acta Cryst. A64, 112-122.

Sheldrick, G. M. (2015). Acta Cryst. C71, 3-8.

Takahashi, D., Oyunzul, L., Onoue, S., Ito, Y., Uchida, S., Simsek, R., Gunduz, M. G., Safak, C. \& Yamada, S. (2008). Biol. Pharm. Bull. 31, 473-479.

Trippier, P. C., Jansen Labby, K., Hawker, D., Mataka, J. \& Silverman, R. (2013). J. Med. Chem. 56, 3121-3147.

Wishart, D. S., Knox, C., Guo, A. C., Shrivastava, S., Hassanali, M., Stothard, P., Chang, Z. \& Woolsey, J. (2006). Nucleic Acids Res. 34, 668-672.

Yang, X. H., Zhang, P. H., Zhou, Y. H., Liu, C. G., Lin, X. Y. \& Cui, J. F. (2011). Arkivoc, x, 327-337.

Zamponi, G. (2005). Voltage-Gated Calcium Channels, pp. 327-337. New York: Landes Bioscience. 


\section{supporting information}

Acta Cryst. (2018). E74, 1417-1420 [https://doi.org/10.1107/S2056989018011982]

Crystal structure of ethyl 4-[4-(dimethylamino) phenyl]-2,7,7-trimethyl-5oxo-1,4,5,6,7,8-hexahydroquinoline-3-carboxylate

\section{Scott A. Steiger, Chun Li and Nicholas R. Natale}

Computing details

Data collection: APEX2 (Bruker, 2013); cell refinement: SAINT (Bruker, 2013); data reduction: SAINT (Bruker, 2013); program(s) used to solve structure: SHELXS97 (Sheldrick, 2008); program(s) used to refine structure: SHELXL2014 (Sheldrick, 2015); molecular graphics: OLEX2 (Dolomanov et al., 2009); software used to prepare material for publication: OLEX2 (Dolomanov et al., 2009).

Ethyl 4-[4-(dimethylamino)phenyl]-2,7,7-trimethyl-5-oxo-1,4,5,6,7,8-hexahydroquinoline-3-carboxylate

\section{Crystal data}

$\mathrm{C}_{23} \mathrm{H}_{30} \mathrm{~N}_{2} \mathrm{O}_{3}$

$M_{r}=382.49$

Monoclinic, $P 2_{1} / n$

$a=9.6834(3) \AA$

$b=18.2390(5) \AA$

$c=12.2073(3) \AA$

$\beta=105.4464(14)^{\circ}$

$V=2078.12(10) \AA^{3}$

$Z=4$

$F(000)=824$

Data collection

\section{Bruker SMART BREEZE CCD}

diffractometer

Radiation source: $2 \mathrm{~kW}$ sealed X-ray tube $\varphi$ and $\omega$ scans

Absorption correction: multi-scan

(SADABS; Bruker, 2013)

$T_{\min }=0.923, T_{\max }=1.000$

48649 measured reflections

\section{Refinement}

Refinement on $F^{2}$

Least-squares matrix: full

$R\left[F^{2}>2 \sigma\left(F^{2}\right)\right]=0.049$

$w R\left(F^{2}\right)=0.142$

$S=1.02$

7211 reflections

264 parameters

0 restraints
$D_{\mathrm{x}}=1.223 \mathrm{Mg} \mathrm{m}^{-3}$

Melting point: $240.1 \mathrm{~K}$

Mo $K \alpha$ radiation, $\lambda=0.71073 \AA$

Cell parameters from 9940 reflections

$\theta=2.5-31.8^{\circ}$

$\mu=0.08 \mathrm{~mm}^{-1}$

$T=100 \mathrm{~K}$

Prism, yellow

$0.46 \times 0.30 \times 0.26 \mathrm{~mm}$

7211 independent reflections

5703 reflections with $I>2 \sigma(I)$

$R_{\text {int }}=0.038$

$\theta_{\max }=32.1^{\circ}, \theta_{\min }=2.4^{\circ}$

$h=-13 \rightarrow 14$

$k=-27 \rightarrow 27$

$l=-18 \rightarrow 18$

Primary atom site location: structure-invariant direct methods

Hydrogen site location: mixed

$\mathrm{H}$ atoms treated by a mixture of independent and constrained refinement

$w=1 /\left[\sigma^{2}\left(F_{\mathrm{o}}^{2}\right)+(0.0858 P)^{2}+0.4034 P\right]$ where $P=\left(F_{\mathrm{o}}{ }^{2}+2 F_{\mathrm{c}}{ }^{2}\right) / 3$

$(\Delta / \sigma)_{\max }<0.001$

$\Delta \rho_{\max }=0.56 \mathrm{e} \AA^{-3}$

$\Delta \rho_{\min }=-0.21 \mathrm{e} \AA^{-3}$ 


\section{Special details}

Geometry. All esds (except the esd in the dihedral angle between two 1.s. planes) are estimated using the full covariance matrix. The cell esds are taken into account individually in the estimation of esds in distances, angles and torsion angles; correlations between esds in cell parameters are only used when they are defined by crystal symmetry. An approximate (isotropic) treatment of cell esds is used for estimating esds involving l.s. planes.

Fractional atomic coordinates and isotropic or equivalent isotropic displacement parameters $\left(\hat{A}^{2}\right)$

\begin{tabular}{|c|c|c|c|c|}
\hline & $x$ & $y$ & $z$ & $U_{\text {iso }} * / U_{\text {eq }}$ \\
\hline $\mathrm{O} 1$ & $0.56627(8)$ & $0.18519(4)$ & $0.65486(6)$ & $0.02329(16)$ \\
\hline $\mathrm{O} 2$ & $0.20382(10)$ & $0.48113(4)$ & $0.62250(7)$ & $0.0315(2)$ \\
\hline $\mathrm{O} 3$ & $0.32826(8)$ & $0.40562(4)$ & $0.75768(6)$ & $0.02233(16)$ \\
\hline N1 & $0.18215(9)$ & $0.30161(5)$ & $0.39338(7)$ & $0.01757(16)$ \\
\hline $\mathrm{N} 2$ & $0.02043(10)$ & $0.12538(5)$ & $0.88241(7)$ & 0.02398 (19) \\
\hline $\mathrm{C} 2$ & $0.17423(10)$ & $0.35953(5)$ & $0.46481(8)$ & $0.01733(18)$ \\
\hline $\mathrm{C} 3$ & $0.25415(10)$ & $0.35787(5)$ & $0.57458(8)$ & $0.01643(17)$ \\
\hline $\mathrm{C} 4$ & $0.33616(10)$ & $0.28901(5)$ & $0.62517(7)$ & $0.01576(17)$ \\
\hline $\mathrm{H} 4$ & 0.4296 & 0.3045 & 0.6776 & $0.019^{*}$ \\
\hline $\mathrm{C} 5$ & $0.48613(10)$ & $0.19197(5)$ & $0.55790(8)$ & $0.01629(17)$ \\
\hline C6 & $0.51589(10)$ & $0.14782(6)$ & $0.46162(8)$ & $0.01877(18)$ \\
\hline H6A & 0.5626 & 0.1012 & 0.4928 & $0.023^{*}$ \\
\hline H6B & 0.5842 & 0.1754 & 0.4297 & $0.023^{*}$ \\
\hline $\mathrm{C} 7$ & $0.38262(11)$ & $0.13022(6)$ & $0.36546(8)$ & $0.01950(19)$ \\
\hline $\mathrm{C} 8$ & $0.30521(11)$ & $0.20264(6)$ & $0.32603(8)$ & $0.01878(18)$ \\
\hline H8A & 0.3603 & 0.2306 & 0.2824 & $0.023^{*}$ \\
\hline H8B & 0.2098 & 0.1919 & 0.2744 & $0.023^{*}$ \\
\hline C9 & $0.28658(10)$ & $0.24911(5)$ & $0.42229(8)$ & $0.01549(17)$ \\
\hline $\mathrm{C} 10$ & $0.36785(10)$ & $0.24286(5)$ & $0.53149(7)$ & $0.01521(17)$ \\
\hline $\mathrm{C} 11$ & $0.28490(13)$ & $0.07753(6)$ & $0.40759(11)$ & $0.0284(2)$ \\
\hline H11A & 0.2589 & 0.0993 & 0.4727 & $0.043^{*}$ \\
\hline H11B & 0.3353 & 0.0311 & 0.4306 & $0.043^{*}$ \\
\hline $\mathrm{H} 11 \mathrm{C}$ & 0.1979 & 0.0684 & 0.3464 & $0.043^{*}$ \\
\hline $\mathrm{C} 12$ & $0.42649(14)$ & $0.09541(8)$ & $0.26599(10)$ & $0.0327(3)$ \\
\hline $\mathrm{H} 12 \mathrm{~A}$ & 0.4741 & 0.0484 & 0.2901 & $0.049^{*}$ \\
\hline H12B & 0.4924 & 0.1282 & 0.2412 & $0.049^{*}$ \\
\hline $\mathrm{H} 12 \mathrm{C}$ & 0.3411 & 0.0872 & 0.2029 & $0.049^{*}$ \\
\hline $\mathrm{C} 13$ & $0.07190(12)$ & $0.41868(6)$ & $0.40825(9)$ & $0.0234(2)$ \\
\hline $\mathrm{H} 13 \mathrm{~A}$ & 0.0221 & 0.4034 & 0.3309 & $0.035^{*}$ \\
\hline H13B & 0.1252 & 0.4640 & 0.4053 & $0.035^{*}$ \\
\hline $\mathrm{H} 13 \mathrm{C}$ & 0.0017 & 0.4273 & 0.4517 & $0.035^{*}$ \\
\hline $\mathrm{C} 14$ & $0.25628(11)$ & $0.42135(5)$ & $0.64921(8)$ & $0.01880(18)$ \\
\hline $\mathrm{C} 15$ & $0.32585(12)$ & $0.46169(6)$ & $0.84080(9)$ & $0.0225(2)$ \\
\hline $\mathrm{H} 15 \mathrm{~A}$ & 0.2268 & 0.4792 & 0.8323 & $0.027^{*}$ \\
\hline H15B & 0.3860 & 0.5039 & 0.8316 & $0.027^{*}$ \\
\hline $\mathrm{C} 16$ & $0.38454(12)$ & $0.42620(6)$ & $0.95533(9)$ & $0.0232(2)$ \\
\hline H16A & 0.3267 & 0.3831 & 0.9614 & $0.035^{*}$ \\
\hline H16B & 0.3811 & 0.4613 & 1.0153 & $0.035^{*}$ \\
\hline $\mathrm{H} 16 \mathrm{C}$ & 0.4840 & 0.4112 & 0.9637 & $0.035^{*}$ \\
\hline
\end{tabular}




$\begin{array}{lllll}\text { C17 } & 0.25404(10) & 0.24552(5) & 0.69388(8) & 0.01645(17) \\ \text { C18 } & 0.12125(11) & 0.21446(6) & 0.64193(8) & 0.02084(19) \\ \text { H18 } & 0.0818 & 0.2208 & 0.5625 & 0.025^{*} \\ \text { C19 } & 0.04507(12) & 0.17457(6) & 0.70303(8) & 0.0224(2) \\ \text { H19 } & -0.0452 & 0.1543 & 0.6648 & 0.027^{*} \\ \text { C20 } & 0.09936(11) & 0.16371(5) & 0.82071(8) & 0.01958(19) \\ \text { C21 } & 0.23303(11) & 0.19489(6) & 0.87312(8) & 0.0213(2) \\ \text { H21 } & 0.2733 & 0.1884 & 0.9524 & 0.026^{*} \\ \text { C22 } & 0.30727(11) & 0.23512(6) & 0.81049(8) & 0.01973(19) \\ \text { H22 } & 0.3970 & 0.2561 & 0.8484 & 0.024^{*} \\ \text { C23 } & -0.08672(14) & 0.07366(7) & 0.82229(10) & 0.0311(2) \\ \text { H23A } & -0.1338(9) & 0.0513(5) & 0.8755(5) & 0.047^{*} \\ \text { H23B } & -0.0408(5) & 0.0356(5) & 0.7881(8) & 0.047^{*} \\ \text { H23C } & -0.1578(9) & 0.0993(3) & 0.7628(8) & 0.047^{*} \\ \text { C24 } & 0.09169(14) & 0.10629(7) & 0.99913(9) & 0.0295(2) \\ \text { H24A } & 0.0239 & 0.0812 & 1.0333 & 0.044^{*} \\ \text { H24B } & 0.1265 & 0.1510 & 1.0422 & 0.044^{*} \\ \text { H24C } & 0.1728 & 0.0738 & 1.0007 & 0.044^{*} \\ \text { H1 } & 0.1367(16) & 0.3063(8) & 0.3211(13) & 0.029(4) *\end{array}$

Atomic displacement parameters $\left(\AA^{2}\right)$

\begin{tabular}{lllllll}
\hline & $U^{11}$ & $U^{22}$ & $U^{33}$ & $U^{12}$ & $U^{13}$ & $U^{23}$ \\
\hline $\mathrm{O} 1$ & $0.0214(3)$ & $0.0261(4)$ & $0.0175(3)$ & $0.0058(3)$ & $-0.0032(3)$ & $-0.0014(3)$ \\
$\mathrm{O} 2$ & $0.0422(5)$ & $0.0195(4)$ & $0.0272(4)$ & $0.0107(3)$ & $-0.0006(3)$ & $-0.0023(3)$ \\
$\mathrm{O} 3$ & $0.0275(4)$ & $0.0178(3)$ & $0.0185(3)$ & $0.0034(3)$ & $0.0006(3)$ & $-0.0048(2)$ \\
$\mathrm{N} 1$ & $0.0173(4)$ & $0.0179(4)$ & $0.0144(3)$ & $0.0033(3)$ & $-0.0012(3)$ & $0.0002(3)$ \\
$\mathrm{N} 2$ & $0.0277(5)$ & $0.0273(5)$ & $0.0197(4)$ & $0.0008(4)$ & $0.0111(3)$ & $0.0017(3)$ \\
$\mathrm{C} 2$ & $0.0162(4)$ & $0.0156(4)$ & $0.0188(4)$ & $0.0010(3)$ & $0.0021(3)$ & $0.0005(3)$ \\
$\mathrm{C} 3$ & $0.0162(4)$ & $0.0149(4)$ & $0.0170(4)$ & $0.0013(3)$ & $0.0024(3)$ & $-0.0007(3)$ \\
$\mathrm{C} 4$ & $0.0160(4)$ & $0.0153(4)$ & $0.0139(4)$ & $0.0011(3)$ & $0.0004(3)$ & $-0.0007(3)$ \\
$\mathrm{C} 5$ & $0.0149(4)$ & $0.0160(4)$ & $0.0166(4)$ & $0.0001(3)$ & $0.0019(3)$ & $-0.0002(3)$ \\
$\mathrm{C} 6$ & $0.0150(4)$ & $0.0217(4)$ & $0.0185(4)$ & $0.0032(3)$ & $0.0024(3)$ & $-0.0015(3)$ \\
$\mathrm{C} 7$ & $0.0174(4)$ & $0.0210(4)$ & $0.0184(4)$ & $0.0027(3)$ & $0.0019(3)$ & $-0.0040(3)$ \\
$\mathrm{C} 8$ & $0.0191(4)$ & $0.0215(4)$ & $0.0141(4)$ & $0.0026(3)$ & $0.0016(3)$ & $-0.0018(3)$ \\
$\mathrm{C} 9$ & $0.0146(4)$ & $0.0156(4)$ & $0.0153(4)$ & $0.0004(3)$ & $0.0023(3)$ & $0.0008(3)$ \\
$\mathrm{C} 10$ & $0.0154(4)$ & $0.0147(4)$ & $0.0143(4)$ & $0.0006(3)$ & $0.0019(3)$ & $0.0004(3)$ \\
$\mathrm{C} 11$ & $0.0264(5)$ & $0.0193(5)$ & $0.0365(6)$ & $-0.0039(4)$ & $0.0034(4)$ & $-0.0031(4)$ \\
$\mathrm{C} 12$ & $0.0304(6)$ & $0.0408(7)$ & $0.0234(5)$ & $0.0129(5)$ & $0.0013(4)$ & $-0.0119(4)$ \\
$\mathrm{C} 13$ & $0.0216(5)$ & $0.0202(5)$ & $0.0244(5)$ & $0.0064(4)$ & $-0.0008(4)$ & $0.0019(4)$ \\
C14 & $0.0173(4)$ & $0.0180(4)$ & $0.0199(4)$ & $0.0006(3)$ & $0.0027(3)$ & $-0.0015(3)$ \\
C15 & $0.0243(5)$ & $0.0191(4)$ & $0.0227(4)$ & $0.0003(4)$ & $0.0036(4)$ & $-0.0073(3)$ \\
C16 & $0.0222(5)$ & $0.0260(5)$ & $0.0215(4)$ & $-0.0034(4)$ & $0.0059(4)$ & $-0.0051(4)$ \\
C17 & $0.0186(4)$ & $0.0153(4)$ & $0.0146(4)$ & $0.0022(3)$ & $0.0028(3)$ & $-0.0015(3)$ \\
C18 & $0.0236(5)$ & $0.0236(5)$ & $0.0140(4)$ & $-0.0035(4)$ & $0.0028(3)$ & $-0.0013(3)$ \\
C19 & $0.0242(5)$ & $0.0256(5)$ & $0.0174(4)$ & $-0.0043(4)$ & $0.0055(4)$ & $-0.0021(3)$ \\
C20 & $0.0242(5)$ & $0.0181(4)$ & $0.0181(4)$ & $0.0033(4)$ & $0.0087(4)$ & $-0.0001(3)$ \\
C21 & $0.0237(5)$ & $0.0252(5)$ & $0.0143(4)$ & $0.0044(4)$ & $0.0037(3)$ & $0.0016(3)$ \\
& & & & & &
\end{tabular}




\begin{tabular}{lllllll}
$\mathrm{C} 22$ & $0.0192(4)$ & $0.0220(5)$ & $0.0157(4)$ & $0.0026(3)$ & $0.0007(3)$ & $0.0001(3)$ \\
$\mathrm{C} 23$ & $0.0338(6)$ & $0.0328(6)$ & $0.0318(6)$ & $-0.0069(5)$ & $0.0173(5)$ & $-0.0015(4)$ \\
$\mathrm{C} 24$ & $0.0344(6)$ & $0.0346(6)$ & $0.0225(5)$ & $0.0059(5)$ & $0.0129(4)$ & $0.0078(4)$ \\
\hline
\end{tabular}

Geometric parameters $\left(\AA,{ }^{\circ}\right)$

\begin{tabular}{|c|c|c|c|}
\hline $\mathrm{O} 1-\mathrm{C} 5$ & $1.2367(11)$ & $\mathrm{C} 11-\mathrm{H} 11 \mathrm{~B}$ & 0.9800 \\
\hline $\mathrm{O} 2-\mathrm{C} 14$ & $1.2103(12)$ & $\mathrm{C} 11-\mathrm{H} 11 \mathrm{C}$ & 0.9800 \\
\hline $\mathrm{O} 3-\mathrm{C} 14$ & $1.3527(12)$ & $\mathrm{C} 12-\mathrm{H} 12 \mathrm{~A}$ & 0.9800 \\
\hline $\mathrm{O} 3-\mathrm{C} 15$ & $1.4451(12)$ & $\mathrm{C} 12-\mathrm{H} 12 \mathrm{~B}$ & 0.9800 \\
\hline $\mathrm{N} 1-\mathrm{C} 2$ & $1.3849(12)$ & $\mathrm{C} 12-\mathrm{H} 12 \mathrm{C}$ & 0.9800 \\
\hline $\mathrm{N} 1-\mathrm{C} 9$ & $1.3690(12)$ & $\mathrm{C} 13-\mathrm{H} 13 \mathrm{~A}$ & 0.9800 \\
\hline $\mathrm{N} 1-\mathrm{H} 1$ & $0.879(15)$ & $\mathrm{C} 13-\mathrm{H} 13 \mathrm{~B}$ & 0.9800 \\
\hline $\mathrm{N} 2-\mathrm{C} 20$ & $1.3953(13)$ & $\mathrm{C} 13-\mathrm{H} 13 \mathrm{C}$ & 0.9800 \\
\hline $\mathrm{N} 2-\mathrm{C} 23$ & $1.4489(16)$ & $\mathrm{C} 15-\mathrm{H} 15 \mathrm{~A}$ & 0.9900 \\
\hline $\mathrm{N} 2-\mathrm{C} 24$ & $1.4505(14)$ & $\mathrm{C} 15-\mathrm{H} 15 \mathrm{~B}$ & 0.9900 \\
\hline $\mathrm{C} 2-\mathrm{C} 3$ & $1.3575(12)$ & $\mathrm{C} 15-\mathrm{C} 16$ & $1.5078(15)$ \\
\hline $\mathrm{C} 2-\mathrm{C} 13$ & $1.5028(13)$ & C16-H16A & 0.9800 \\
\hline $\mathrm{C} 3-\mathrm{C} 4$ & $1.5259(13)$ & $\mathrm{C} 16-\mathrm{H} 16 \mathrm{~B}$ & 0.9800 \\
\hline $\mathrm{C} 3-\mathrm{C} 14$ & $1.4700(13)$ & $\mathrm{C} 16-\mathrm{H} 16 \mathrm{C}$ & 0.9800 \\
\hline $\mathrm{C} 4-\mathrm{H} 4$ & 1.0000 & $\mathrm{C} 17-\mathrm{C} 18$ & $1.3936(14)$ \\
\hline $\mathrm{C} 4-\mathrm{C} 10$ & $1.5157(13)$ & $\mathrm{C} 17-\mathrm{C} 22$ & $1.3917(13)$ \\
\hline $\mathrm{C} 4-\mathrm{C} 17$ & $1.5233(13)$ & $\mathrm{C} 18-\mathrm{H} 18$ & 0.9500 \\
\hline $\mathrm{C} 5-\mathrm{C} 6$ & $1.5143(13)$ & $\mathrm{C} 18-\mathrm{C} 19$ & $1.3867(14)$ \\
\hline $\mathrm{C} 5-\mathrm{C} 10$ & $1.4425(13)$ & $\mathrm{C} 19-\mathrm{H} 19$ & 0.9500 \\
\hline C6-H6A & 0.9900 & $\mathrm{C} 19-\mathrm{C} 20$ & $1.4061(14)$ \\
\hline $\mathrm{C} 6-\mathrm{H} 6 \mathrm{~B}$ & 0.9900 & $\mathrm{C} 20-\mathrm{C} 21$ & $1.4024(15)$ \\
\hline $\mathrm{C} 6-\mathrm{C} 7$ & $1.5296(13)$ & $\mathrm{C} 21-\mathrm{H} 21$ & 0.9500 \\
\hline $\mathrm{C} 7-\mathrm{C} 8$ & $1.5317(14)$ & $\mathrm{C} 21-\mathrm{C} 22$ & $1.3903(14)$ \\
\hline $\mathrm{C} 7-\mathrm{C} 11$ & $1.5306(16)$ & $\mathrm{C} 22-\mathrm{H} 22$ & 0.9500 \\
\hline $\mathrm{C} 7-\mathrm{C} 12$ & $1.5272(15)$ & $\mathrm{C} 23-\mathrm{H} 23 \mathrm{~A}$ & $0.976(10)$ \\
\hline $\mathrm{C} 8-\mathrm{H} 8 \mathrm{~A}$ & 0.9900 & $\mathrm{C} 23-\mathrm{H} 23 \mathrm{~B}$ & $0.976(10)$ \\
\hline $\mathrm{C} 8-\mathrm{H} 8 \mathrm{~B}$ & 0.9900 & $\mathrm{C} 23-\mathrm{H} 23 \mathrm{C}$ & $0.976(10)$ \\
\hline $\mathrm{C} 8-\mathrm{C} 9$ & $1.4982(13)$ & $\mathrm{C} 24-\mathrm{H} 24 \mathrm{~A}$ & 0.9800 \\
\hline $\mathrm{C} 9-\mathrm{C} 10$ & $1.3603(12)$ & $\mathrm{C} 24-\mathrm{H} 24 \mathrm{~B}$ & 0.9800 \\
\hline $\mathrm{C} 11-\mathrm{H} 11 \mathrm{~A}$ & 0.9800 & $\mathrm{C} 24-\mathrm{H} 24 \mathrm{C}$ & 0.9800 \\
\hline $\mathrm{C} 14-\mathrm{O} 3-\mathrm{C} 15$ & $115.89(8)$ & $\mathrm{H} 12 \mathrm{~A}-\mathrm{C} 12-\mathrm{H} 12 \mathrm{~B}$ & 109.5 \\
\hline $\mathrm{C} 2-\mathrm{N} 1-\mathrm{H} 1$ & $117.4(10)$ & $\mathrm{H} 12 \mathrm{~A}-\mathrm{C} 12-\mathrm{H} 12 \mathrm{C}$ & 109.5 \\
\hline $\mathrm{C} 9-\mathrm{N} 1-\mathrm{C} 2$ & $122.15(8)$ & $\mathrm{H} 12 \mathrm{~B}-\mathrm{C} 12-\mathrm{H} 12 \mathrm{C}$ & 109.5 \\
\hline $\mathrm{C} 9-\mathrm{N} 1-\mathrm{H} 1$ & $117.7(10)$ & $\mathrm{C} 2-\mathrm{C} 13-\mathrm{H} 13 \mathrm{~A}$ & 109.5 \\
\hline $\mathrm{C} 20-\mathrm{N} 2-\mathrm{C} 23$ & $118.30(9)$ & $\mathrm{C} 2-\mathrm{C} 13-\mathrm{H} 13 \mathrm{~B}$ & 109.5 \\
\hline $\mathrm{C} 20-\mathrm{N} 2-\mathrm{C} 24$ & $117.70(10)$ & $\mathrm{C} 2-\mathrm{C} 13-\mathrm{H} 13 \mathrm{C}$ & 109.5 \\
\hline $\mathrm{C} 23-\mathrm{N} 2-\mathrm{C} 24$ & $115.49(9)$ & $\mathrm{H} 13 \mathrm{~A}-\mathrm{C} 13-\mathrm{H} 13 \mathrm{~B}$ & 109.5 \\
\hline $\mathrm{N} 1-\mathrm{C} 2-\mathrm{C} 13$ & $113.49(8)$ & $\mathrm{H} 13 \mathrm{~A}-\mathrm{C} 13-\mathrm{H} 13 \mathrm{C}$ & 109.5 \\
\hline $\mathrm{C} 3-\mathrm{C} 2-\mathrm{N} 1$ & $119.47(8)$ & $\mathrm{H} 13 \mathrm{~B}-\mathrm{C} 13-\mathrm{H} 13 \mathrm{C}$ & 109.5 \\
\hline $\mathrm{C} 3-\mathrm{C} 2-\mathrm{C} 13$ & $127.03(9)$ & $\mathrm{O} 2-\mathrm{C} 14-\mathrm{O} 3$ & $121.65(9)$ \\
\hline $\mathrm{C} 2-\mathrm{C} 3-\mathrm{C} 4$ & $121.07(8)$ & $\mathrm{O} 2-\mathrm{C} 14-\mathrm{C} 3$ & $127.36(9)$ \\
\hline
\end{tabular}




\begin{tabular}{|c|c|c|c|}
\hline $\mathrm{C} 2-\mathrm{C} 3-\mathrm{C} 14$ & $120.30(9)$ & $\mathrm{O} 3-\mathrm{C} 14-\mathrm{C} 3$ & $110.99(8)$ \\
\hline $\mathrm{C} 14-\mathrm{C} 3-\mathrm{C} 4$ & $118.53(8)$ & $\mathrm{O} 3-\mathrm{C} 15-\mathrm{H} 15 \mathrm{~A}$ & 110.5 \\
\hline $\mathrm{C} 3-\mathrm{C} 4-\mathrm{H} 4$ & 108.1 & $\mathrm{O} 3-\mathrm{C} 15-\mathrm{H} 15 \mathrm{~B}$ & 110.5 \\
\hline $\mathrm{C} 10-\mathrm{C} 4-\mathrm{C} 3$ & $109.87(7)$ & $\mathrm{O} 3-\mathrm{C} 15-\mathrm{C} 16$ & $105.94(8)$ \\
\hline $\mathrm{C} 10-\mathrm{C} 4-\mathrm{H} 4$ & 108.1 & $\mathrm{H} 15 \mathrm{~A}-\mathrm{C} 15-\mathrm{H} 15 \mathrm{~B}$ & 108.7 \\
\hline $\mathrm{C} 10-\mathrm{C} 4-\mathrm{C} 17$ & $111.50(7)$ & $\mathrm{C} 16-\mathrm{C} 15-\mathrm{H} 15 \mathrm{~A}$ & 110.5 \\
\hline $\mathrm{C} 17-\mathrm{C} 4-\mathrm{C} 3$ & $111.08(8)$ & $\mathrm{C} 16-\mathrm{C} 15-\mathrm{H} 15 \mathrm{~B}$ & 110.5 \\
\hline $\mathrm{C} 17-\mathrm{C} 4-\mathrm{H} 4$ & 108.1 & $\mathrm{C} 15-\mathrm{C} 16-\mathrm{H} 16 \mathrm{~A}$ & 109.5 \\
\hline $\mathrm{O} 1-\mathrm{C} 5-\mathrm{C} 6$ & $119.28(8)$ & $\mathrm{C} 15-\mathrm{C} 16-\mathrm{H} 16 \mathrm{~B}$ & 109.5 \\
\hline $\mathrm{O} 1-\mathrm{C} 5-\mathrm{C} 10$ & $122.46(9)$ & $\mathrm{C} 15-\mathrm{C} 16-\mathrm{H} 16 \mathrm{C}$ & 109.5 \\
\hline $\mathrm{C} 10-\mathrm{C} 5-\mathrm{C} 6$ & $118.20(8)$ & $\mathrm{H} 16 \mathrm{~A}-\mathrm{C} 16-\mathrm{H} 16 \mathrm{~B}$ & 109.5 \\
\hline $\mathrm{C} 5-\mathrm{C} 6-\mathrm{H} 6 \mathrm{~A}$ & 108.7 & $\mathrm{H} 16 \mathrm{~A}-\mathrm{C} 16-\mathrm{H} 16 \mathrm{C}$ & 109.5 \\
\hline $\mathrm{C} 5-\mathrm{C} 6-\mathrm{H} 6 \mathrm{~B}$ & 108.7 & $\mathrm{H} 16 \mathrm{~B}-\mathrm{C} 16-\mathrm{H} 16 \mathrm{C}$ & 109.5 \\
\hline $\mathrm{C} 5-\mathrm{C} 6-\mathrm{C} 7$ & $114.28(8)$ & $\mathrm{C} 18-\mathrm{C} 17-\mathrm{C} 4$ & $120.98(8)$ \\
\hline $\mathrm{H} 6 \mathrm{~A}-\mathrm{C} 6-\mathrm{H} 6 \mathrm{~B}$ & 107.6 & $\mathrm{C} 22-\mathrm{C} 17-\mathrm{C} 4$ & $121.92(9)$ \\
\hline $\mathrm{C} 7-\mathrm{C} 6-\mathrm{H} 6 \mathrm{~A}$ & 108.7 & $\mathrm{C} 22-\mathrm{C} 17-\mathrm{C} 18$ & $117.10(9)$ \\
\hline $\mathrm{C} 7-\mathrm{C} 6-\mathrm{H} 6 \mathrm{~B}$ & 108.7 & $\mathrm{C} 17-\mathrm{C} 18-\mathrm{H} 18$ & 119.1 \\
\hline $\mathrm{C} 6-\mathrm{C} 7-\mathrm{C} 8$ & $107.60(8)$ & $\mathrm{C} 19-\mathrm{C} 18-\mathrm{C} 17$ & $121.80(9)$ \\
\hline $\mathrm{C} 6-\mathrm{C} 7-\mathrm{C} 11$ & $110.16(9)$ & $\mathrm{C} 19-\mathrm{C} 18-\mathrm{H} 18$ & 119.1 \\
\hline $\mathrm{C} 11-\mathrm{C} 7-\mathrm{C} 8$ & $110.63(9)$ & $\mathrm{C} 18-\mathrm{C} 19-\mathrm{H} 19$ & 119.5 \\
\hline $\mathrm{C} 12-\mathrm{C} 7-\mathrm{C} 6$ & $109.94(9)$ & $\mathrm{C} 18-\mathrm{C} 19-\mathrm{C} 20$ & $121.05(10)$ \\
\hline $\mathrm{C} 12-\mathrm{C} 7-\mathrm{C} 8$ & $108.97(9)$ & $\mathrm{C} 20-\mathrm{C} 19-\mathrm{H} 19$ & 119.5 \\
\hline $\mathrm{C} 12-\mathrm{C} 7-\mathrm{C} 11$ & $109.50(9)$ & $\mathrm{N} 2-\mathrm{C} 20-\mathrm{C} 19$ & $120.83(10)$ \\
\hline $\mathrm{C} 7-\mathrm{C} 8-\mathrm{H} 8 \mathrm{~A}$ & 109.0 & $\mathrm{~N} 2-\mathrm{C} 20-\mathrm{C} 21$ & $121.91(9)$ \\
\hline $\mathrm{C} 7-\mathrm{C} 8-\mathrm{H} 8 \mathrm{~B}$ & 109.0 & $\mathrm{C} 21-\mathrm{C} 20-\mathrm{C} 19$ & $117.22(9)$ \\
\hline $\mathrm{H} 8 \mathrm{~A}-\mathrm{C} 8-\mathrm{H} 8 \mathrm{~B}$ & 107.8 & $\mathrm{C} 20-\mathrm{C} 21-\mathrm{H} 21$ & 119.6 \\
\hline $\mathrm{C} 9-\mathrm{C} 8-\mathrm{C} 7$ & $113.11(8)$ & $\mathrm{C} 22-\mathrm{C} 21-\mathrm{C} 20$ & $120.85(9)$ \\
\hline $\mathrm{C} 9-\mathrm{C} 8-\mathrm{H} 8 \mathrm{~A}$ & 109.0 & $\mathrm{C} 22-\mathrm{C} 21-\mathrm{H} 21$ & 119.6 \\
\hline $\mathrm{C} 9-\mathrm{C} 8-\mathrm{H} 8 \mathrm{~B}$ & 109.0 & $\mathrm{C} 17-\mathrm{C} 22-\mathrm{H} 22$ & 119.0 \\
\hline $\mathrm{N} 1-\mathrm{C} 9-\mathrm{C} 8$ & $115.37(8)$ & $\mathrm{C} 21-\mathrm{C} 22-\mathrm{C} 17$ & $121.97(9)$ \\
\hline $\mathrm{C} 10-\mathrm{C} 9-\mathrm{N} 1$ & $120.40(8)$ & $\mathrm{C} 21-\mathrm{C} 22-\mathrm{H} 22$ & 119.0 \\
\hline $\mathrm{C} 10-\mathrm{C} 9-\mathrm{C} 8$ & $124.20(8)$ & $\mathrm{N} 2-\mathrm{C} 23-\mathrm{H} 23 \mathrm{~A}$ & 109.5 \\
\hline $\mathrm{C} 5-\mathrm{C} 10-\mathrm{C} 4$ & $119.80(8)$ & $\mathrm{N} 2-\mathrm{C} 23-\mathrm{H} 23 \mathrm{~B}$ & 109.5 \\
\hline $\mathrm{C} 9-\mathrm{C} 10-\mathrm{C} 4$ & $120.82(8)$ & $\mathrm{N} 2-\mathrm{C} 23-\mathrm{H} 23 \mathrm{C}$ & 109.5 \\
\hline $\mathrm{C} 9-\mathrm{C} 10-\mathrm{C} 5$ & $119.38(8)$ & $\mathrm{H} 23 \mathrm{~A}-\mathrm{C} 23-\mathrm{H} 23 \mathrm{~B}$ & 109.5 \\
\hline C7-C11-H11A & 109.5 & $\mathrm{H} 23 \mathrm{~A}-\mathrm{C} 23-\mathrm{H} 23 \mathrm{C}$ & 109.4 \\
\hline C7-C11-H11B & 109.5 & $\mathrm{H} 23 \mathrm{~B}-\mathrm{C} 23-\mathrm{H} 23 \mathrm{C}$ & 109.5 \\
\hline $\mathrm{C} 7-\mathrm{C} 11-\mathrm{H} 11 \mathrm{C}$ & 109.5 & $\mathrm{~N} 2-\mathrm{C} 24-\mathrm{H} 24 \mathrm{~A}$ & 109.5 \\
\hline $\mathrm{H} 11 \mathrm{~A}-\mathrm{C} 11-\mathrm{H} 11 \mathrm{~B}$ & 109.5 & $\mathrm{~N} 2-\mathrm{C} 24-\mathrm{H} 24 \mathrm{~B}$ & 109.5 \\
\hline $\mathrm{H} 11 \mathrm{~A}-\mathrm{C} 11-\mathrm{H} 11 \mathrm{C}$ & 109.5 & $\mathrm{~N} 2-\mathrm{C} 24-\mathrm{H} 24 \mathrm{C}$ & 109.5 \\
\hline $\mathrm{H} 11 \mathrm{~B}-\mathrm{C} 11-\mathrm{H} 11 \mathrm{C}$ & 109.5 & $\mathrm{H} 24 \mathrm{~A}-\mathrm{C} 24-\mathrm{H} 24 \mathrm{~B}$ & 109.5 \\
\hline $\mathrm{C} 7-\mathrm{C} 12-\mathrm{H} 12 \mathrm{~A}$ & 109.5 & $\mathrm{H} 24 \mathrm{~A}-\mathrm{C} 24-\mathrm{H} 24 \mathrm{C}$ & 109.5 \\
\hline $\mathrm{C} 7-\mathrm{C} 12-\mathrm{H} 12 \mathrm{~B}$ & 109.5 & $\mathrm{H} 24 \mathrm{~B}-\mathrm{C} 24-\mathrm{H} 24 \mathrm{C}$ & 109.5 \\
\hline $\mathrm{C} 7-\mathrm{C} 12-\mathrm{H} 12 \mathrm{C}$ & 109.5 & & \\
\hline $\mathrm{O} 1-\mathrm{C} 5-\mathrm{C} 6-\mathrm{C} 7$ & $151.47(9)$ & $\mathrm{C} 8-\mathrm{C} 9-\mathrm{C} 10-\mathrm{C} 4$ & $-176.32(9)$ \\
\hline $\mathrm{O} 1-\mathrm{C} 5-\mathrm{C} 10-\mathrm{C} 4$ & $-1.56(14)$ & $\mathrm{C} 8-\mathrm{C} 9-\mathrm{C} 10-\mathrm{C} 5$ & $4.11(14)$ \\
\hline $\mathrm{O} 1-\mathrm{C} 5-\mathrm{C} 10-\mathrm{C} 9$ & $178.01(9)$ & $\mathrm{C} 9-\mathrm{N} 1-\mathrm{C} 2-\mathrm{C} 3$ & $-12.50(14)$ \\
\hline
\end{tabular}


$-8.68(14)$

$175.02(9)$

$5.51(14)$

$-174.06(9)$

$-177.63(9)$

$-164.12(9)$

$14.20(14)$

$24.82(12)$

$-99.03(10)$

$-7.65(17)$

$172.85(9)$

$156.41(8)$

$-23.16(12)$

63.85 (11)

$-115.98(10)$

$175.96(11)$

$-3.55(12)$

179.87 (9)

$-179.42(9)$

$53.46(11)$

$-67.23(11)$

$172.01(9)$

$-178.95(8)$

$0.63(13)$

-48.04 (11)

$-160.24(8)$

$21.50(14)$

$$
\begin{aligned}
& \mathrm{C} 9-\mathrm{N} 1-\mathrm{C} 2-\mathrm{C} 13 \\
& \mathrm{C} 10-\mathrm{C} 4-\mathrm{C} 17-\mathrm{C} 18 \\
& \mathrm{C} 10-\mathrm{C} 4-\mathrm{C} 17-\mathrm{C} 22 \\
& \mathrm{C} 10-\mathrm{C} 5-\mathrm{C} 6-\mathrm{C} 7 \\
& \mathrm{C} 11-\mathrm{C} 7-\mathrm{C} 8-\mathrm{C} 9 \\
& \mathrm{C} 12-\mathrm{C} 7-\mathrm{C} 8-\mathrm{C} 9 \\
& \mathrm{C} 13-\mathrm{C} 2-\mathrm{C} 3-\mathrm{C} 4 \\
& \mathrm{C} 13-\mathrm{C} 2-\mathrm{C} 3-\mathrm{C} 14 \\
& \mathrm{C} 14-\mathrm{O} 3-\mathrm{C} 15-\mathrm{C} 16 \\
& \mathrm{C} 14-\mathrm{C} 3-\mathrm{C} 4-\mathrm{C} 10 \\
& \mathrm{C} 14-\mathrm{C} 3-\mathrm{C} 4-\mathrm{C} 17 \\
& \mathrm{C} 15-\mathrm{O} 3-\mathrm{C} 14-\mathrm{O} 2 \\
& \mathrm{C} 15-\mathrm{O} 3-\mathrm{C} 14-\mathrm{C} 3 \\
& \mathrm{C} 17-\mathrm{C} 4-\mathrm{C} 10-\mathrm{C} 5 \\
& \mathrm{C} 17-\mathrm{C} 4-\mathrm{C} 10-\mathrm{C} 9 \\
& \mathrm{C} 17-\mathrm{C} 18-\mathrm{C} 19-\mathrm{C} 20 \\
& \mathrm{C} 18-\mathrm{C} 17-\mathrm{C} 22-\mathrm{C} 21 \\
& \mathrm{C} 18-\mathrm{C} 19-\mathrm{C} 20-\mathrm{N} 2 \\
& \mathrm{C} 18-\mathrm{C} 19-\mathrm{C} 20-\mathrm{C} 21 \\
& \text { C19-C20-C21-C22 } \\
& \text { C20-C21-C22-C17 } \\
& \text { C22-C17-C18-C19 } \\
& \text { C23-N2-C20-C19 } \\
& \text { C23-N2-C20-C21 } \\
& \text { C24-N2-C20-C19 } \\
& \text { C24-N2-C20-C21 }
\end{aligned}
$$

$168.16(9)$

$-59.06(11)$

$121.10(10)$

$-31.05(12)$

72.35 (11)

$-167.21(9)$

$170.56(10)$

$-5.74(16)$

168.44 (9)

$-158.81(8)$

77.33 (11)

$6.08(15)$

$-174.38(8)$

$-79.98(10)$

$100.45(10)$

-0.10 (16)

0.74 (15)

178.09 (10)

$0.06(15)$

0.38 (15)

$-0.81(16)$

$-0.29(15)$

23.89 (15)

$-158.17(10)$

$170.49(10)$

-11.57 (15)

Hydrogen-bond geometry $\left(\AA,{ }^{\circ}\right)$

\begin{tabular}{lllll}
\hline$D-\mathrm{H} \cdots A$ & $D-\mathrm{H}$ & $\mathrm{H} \cdots A$ & $D \cdots A$ & $D-\mathrm{H} \cdots A$ \\
\hline $\mathrm{N} 1-\mathrm{H} 1 \cdots \mathrm{O} 1^{\mathrm{i}}$ & $0.879(15)$ & $1.968(15)$ & $2.8380(11)$ & $170.6(14)$ \\
$\mathrm{C} 13-\mathrm{H} 13 C \cdots \mathrm{O} 2^{\mathrm{ii}}$ & 0.98 & 2.57 & $3.1741(15)$ & 120 \\
\hline
\end{tabular}

Symmetry codes: (i) $x-1 / 2,-y+1 / 2, z-1 / 2$; (ii) $-x,-y+1,-z+1$. 